\title{
Study of the CMS RPC detector performance in high radiation background conditions
}

\section{Osvaldo Miguel Colin*}

Universidad Iberoamericana (MX)

E-mail: osvaldo.miguel.colin@cern.ch

\section{Salvador Carrillo}

Universidad Iberoamericana (MX)

E-mail: Salvador.Carrillo@cern.ch

\section{Elsa Fabiola Vázquez}

Universidad Iberoamericana (MX)

E-mail: elsa.fabiola.vazquez.valenciadcern.ch

\section{Cristina Oropeza}

Universidad Iberoamericana $(M X)$

E-mail: cristina.oropeza.barreradcern.ch

The Resistive Plate Chamber (RPC) system at the CMS Detector has been operating successfully since the beginning of data taking. The high Large Hadron Collider (LHC) instantaneous luminosity provides an extremely high flux of ionizing particles. The long period of operation (Run-I and Run-II) in a large radiation background conditions, gives the opportunity to study the operational capability of the RPCs and also to predict a data-driven extrapolation of measured parameters like currents and integrated charged.[1] Those results will be compared to the relevant results obtained from the dedicated $R \& D$ study, where a set of test chambers have been irradiated at GIF++ laboratory setup.[2]

The European Physical Society Conference on High Energy Physics

5-12 July

Venice, Italy

\footnotetext{
*Speaker.
} 


\section{Introduction}

The RPC system is divided in two regions: barrel $(0<|\eta|<1.2)$ and endcap $(0.9<|\eta|<1.6)$. The barrel consists of 5 wheels each with 12 sectors, equipped with 4 muon stations. Each endcap is made by 3 iron disks and 4 muon stations of Cathode Strip Chambers and RPCs. The muon stations contain one layer of double-gap RPC each and are divided along the r-coordinate into 3 chambers. [3]

An RPC gap is made by two parallel bakelite plates placed at a distance of $2 \mathrm{~mm}$ and filled with a gas mixture of $96.2 \% \mathrm{C}_{2} \mathrm{H}_{2} \mathrm{~F}_{4}, 3.5 \% \mathrm{i}-\mathrm{C}_{4} \mathrm{H}_{10}$ and $0.3 \%$ of SF6. High-voltage is applied to the outer graphite-coated surface of the bakelite plates in order to have an electric field inside the gas gap able to generate a charge avalanche along the track of an ionizing particle. [3]

The background rate in the $\eta>1.6$ regions is significantly higher, well beyond the limit reached during previous aging tests. $\mathrm{R} \& \mathrm{D}$ to certify the detector performance under such conditions is ongoing. [4]

The RPC currents read by the Detector Control System (DCS) have been integrated in time. For every single RPC HV channel the current in $[\mu \mathrm{A}]$, being constant for a given period, have been multiplied by the length of that period. The area $\mathrm{Q}_{\mathrm{j}}$ as depicted in Fig. 1 is the integrated charge for the period $\mathrm{j}$. The accumulated integrated charge is obtained by summing over all periods when all RPC HV channels have been at working point ( $\alpha=1$ when RPC ON, otherwise $\alpha=0$ ). (See Fig. 1)

The integrated charge in $[\mathrm{C}]$ has been normalized to the RPC gap area in order to obtain the density of the integrated charge in $\left[\mathrm{C} / \mathrm{cm}^{2}\right]$. This procedure has been applied to all p-p and HI-p collision runs, as well as cosmics and commissioning periods. Since the RPC detector was OFF during the first long shutdown period (LS1) the integrated charge was not calculated.

The HV channel offsets and the ohmic contribution of each RPC chamber are not yet subtracted in the present analysis.

Since the integrated charge and its density are proportional by the inverse of the gap areas, in these plots when we speak about the integrated charge we will mean the density of the integrated charge in $\left[\mathrm{C} / \mathrm{cm}^{2}\right]$.

In the endcap regions of CMS the muon rates and background levels are high [4]. The hottest regions of the barrel are located in the furthermost (RB4) and innermost (RB1) stations to the interaction point. (See Fig. 2)

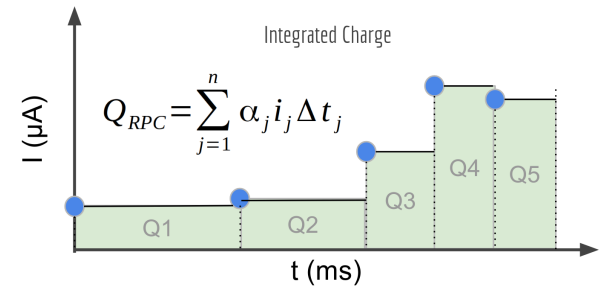

Figure 1: Representation of the currents integrated in time.

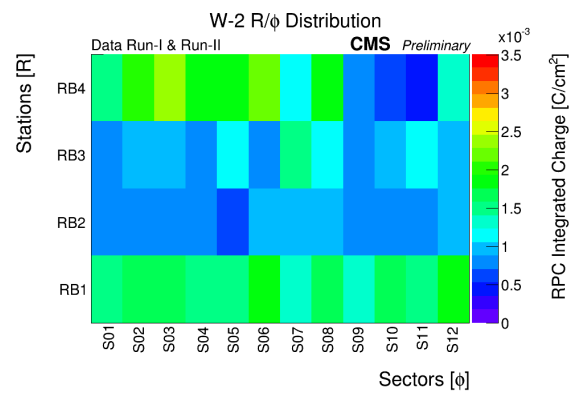

Figure 2: RPC Barrel Integrated Charge Density: $R / \Phi$ Distribution for wheel W-2, Run-I 


\section{RPC Barrel Integrated Charge}

The total integrated charge for the Run-I and Run-II is calculated to be about $1.1 \mathrm{mC} / \mathrm{cm}^{2}$ for the barrel.(See Fig.3) The LS1 period does not contribute to accumulating charge since the RPC detector was OFF. For an extrapolated integrated LHC Luminosity of $3000 \mathrm{fb}^{-1}$, the maximum data-driven extrapolated integrated charge expected in the barrel would be $0.165 \mathrm{C} / \mathrm{cm}^{2}$. (See Fig. 4)

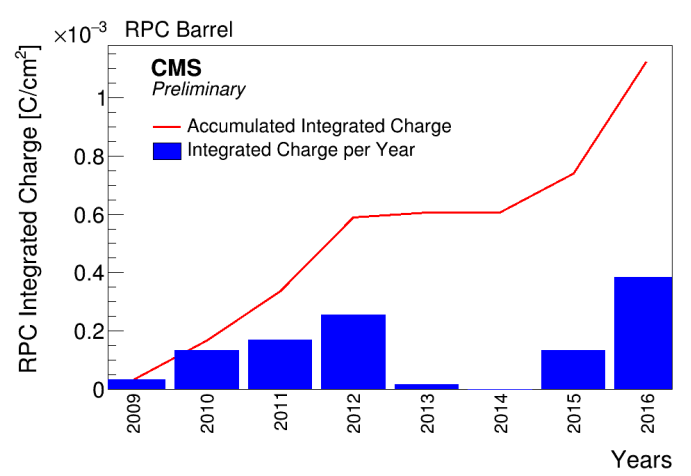

Figure 3: Integrated Charge vs Time - Barrel.The integrated charge per year is shown in blue. The red curve shows the evolution of the accumulated integrated charge in time.

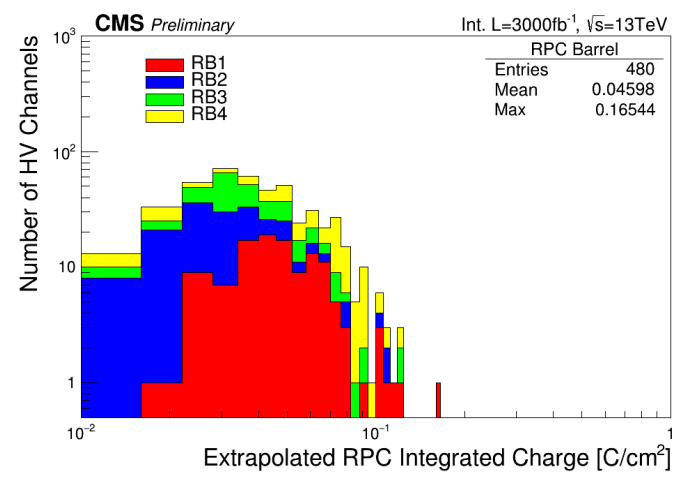

Figure 4: RPC Barrel Extrapolated Integrated Charge at $3000 \mathrm{fb}^{-1}$.

\section{RPC Endcap Integrated Charge}

The total integrated charge for the Run-I and Run-II is calculated to be about $2.7 \mathrm{mC} / \mathrm{cm}^{2}$ for the endcap. (See Fig.5) The LS1 period does not contribute to accumulating charge since the RPC detector was OFF. For an extrapolated integrated LHC Luminosity of $3000 \mathrm{fb}^{-1}$, the maximum data-driven extrapolated integrated charge expected in the endcap would be $0.277 \mathrm{C} / \mathrm{cm}^{2}$. (See Fig. 6)

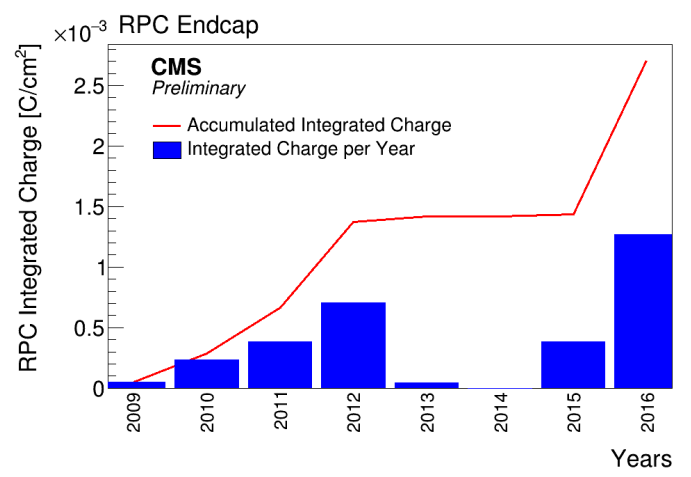

Figure 5: Integrated Charge vs Time - Endcap. The integrated charge per year is shown in blue. The red curve shows the evolution of the accumulated integrated charge in time.

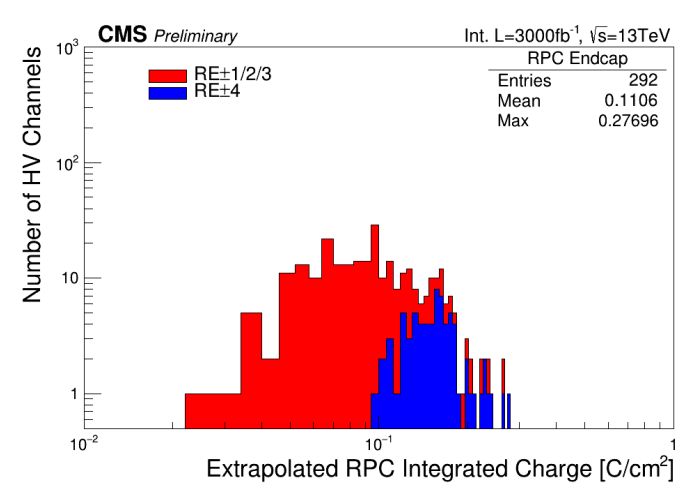

Figure 6: RPC Endcap Extrapolated Integrated Charge at $3000 \mathrm{fb}^{-1}$. 


\section{Conclusions}

The barrel integrates less charge than the endcap. The maximum integrated charge extrapolated at $3000 \mathrm{fb}^{-1}$ for the barrel and the endcap are $165 \mathrm{mC} / \mathrm{cm}^{2}$ and $277 \mathrm{mC} / \mathrm{cm}^{2}$ respectively (no safety factor is included).

The hottest regions are closer to the beam pipeline due to the higher particle flow and at the periphery due to the higher background. (See Fig. 2)

The integrated charge in $\mathrm{RE} \pm 4$ is significantly greater than the rest of the endcap stations. (See Fig. 6)

\section{References}

[1] [CMS Collaboration], CMS, the Compact Muon Solenoid. Muon technical design report, CERN-LHCC-97-32

[2] Aging studies for the CMS RPC system, Poster EPS-HEP2017

[3] G Polese et al 2010 J. Phys.: Conf. Ser. 219022019

[4] The CMS Collaboration et al 2008 JINST 3 S08004 